OPEN ACCESS

Edited by:

Anna Kalbarczyk,

Johns Hopkins University,

United States

Reviewed by:

Piyusha Majumdar,

IIHMR University, India

Abhay Machindra Kudale,

Savitribai Phule Pune University, India

*Correspondence:

Shiau Y. Chong

shiau.chong@unu.edu

Michael J. Penkunas

mike.penkunas@unu.edu

Specialty section:

This article was submitted to

Disease Prevention

and Control Policy,

a section of the journal

Frontiers in Tropical Diseases

Received: 23 August 2021 Accepted: 15 November 2021 Published: 03 December 2021

Citation:

Penkunas MJ, Berdou E, Chong SY, Launois $P$, Rhule ELM and Allotey $P$

(2021) Increasing Knowledge

Translation Capacity in Low- and

Middle-Income Countries: A Model for Implementation Research Training.

Front. Trop. Dis. 2:762966. doi: 10.3389/fitd.2021.762966

\section{Increasing Knowledge Translation Capacity in Low- and Middle-Income Countries: A Model for Implementation Research Training}

\author{
Michael J. Penkunas ${ }^{1 *}$, Evangelia Berdou ${ }^{1,2}$, Shiau Y. Chong ${ }^{1 *}$, Pascal Launois ${ }^{3}$, \\ Emma L. M. Rhule ${ }^{1}$ and Pascale Allotey ${ }^{1}$ \\ 1 United Nations University International Institute for Global Health (UNU-IIGH), Kuala Lumpur, Malaysia, ${ }^{2}$ Data Yarns \\ Research, Brighton, United Kingdom, ${ }^{3}$ Special Programme for Research and Training in Tropical Diseases (TDR), World \\ Health Organization (WHO), Geneva, Switzerland
}

Most health professionals lack the training and expertise to translate clinical innovations into actionable programs. Even though some public health expert communities understand that even widely proven solutions need to be adapted to the demands and characteristics of diverse health systems and societies to be successful, such knowledge has yet to inform routine public health approaches and practices. Therefore, it should not be a surprise that the "know-do" gap between clinical innovations and their on-the-ground application that implementation research seeks to bridge is pervasive and enduring, particularly in low- and middle-income countries. This article draws on a study of implementation research training courses to highlight the various competencies needed to translate different types of knowledge into action, many of which are not adequately addressed in existing curricula. We utilized a four-phase modified Delphi methodology that included a review of the academic and grey literature, one-on-one interviews with experts, virtual dialogue series with key stakeholders, and peer review of the synthesized results. The resulting areas in need of further development include the ability of learners to work as part of a multidisciplinary team, engage various stakeholders, and communicate research findings to decision-makers. Based on these insights, it is argued that knowledge translation in implementation research is a multi-faceted, multi-level sensemaking and communication activity that takes place throughout the research and research-to policy-processes.

Keywords: training, implementation research, capacity building, knowledge translation, low- and middleincome countries

\section{INTRODUCTION}

The academic literature describing efficacious interventions for the treatment and prevention of neglected tropical diseases (NTDs) is well established and showcases several programs with great promise. Mass drug administration for schistosomiasis (1-3) and onchocerciasis (4) are two notable examples where a body of evidence exists supporting its efficacy in treating and 
preventing infection. Yet, contextually specific environmental, geographical, social and demographic factors complicate progress towards elimination and eradication. Even with the availability of efficacious treatment and control techniques, public interest and political backing, an additional effort has to be expended to translate research findings into action to accelerate large scale efforts $(5,6)$.

Researchers' and health practitioners' ability to adapt proven interventions to the requirements of diverse health systems remains hampered by an inadequate capacity to utilize research findings to improve intervention implementation. With a few notable exceptions (7), the "know-do" gap in health service delivery has proven both pervasive and enduring. This disconnect is particularly hard to overcome in low- and middle-income countries (LMICs), where health systems typically lack the human resources to effectively collect, analyze, and interpret locally collected data (8). With efficacious drugs for the treatment and prevention of diseases comes the need to understand how these interventions can be introduced and scaled for the greatest impact within a particular context. The success or failure of disease control efforts is dependent on a range of factors, including the characteristics of the intervention itself, needs of the community, compatibility of the intervention with the health system, engagement with the community, and buy-in from leadership (9). These considerations are context-dependent and, without appropriate examination, can severely limit the scale-up and real-world impact of an efficacious intervention.

Implementation research (IR) in health investigates questions of which interventions work and why (10). IR is further concerned with designing and introducing solutions into health systems addressing those factors impeding implementation and how to promote the scaling and sustainability of successful solutions. The untapped value of IR lies in its ability to help health systems use evidence from "real world" settings to develop and test approaches to improve program implementation. IR is powerful when considering the suite of efficacious interventions with solid evidence bases that fail to perform as expected when introduced into national health systems.

Reframing the IR cycle by - engaging stakeholders, identifying bottlenecks, enacting solutions, evaluating change as a knowledge translation process can be illuminating. However, the overarching knowledge-to-action process is complex and involves the mobilization and utilization of different types of knowledge, at different levels of practice and action. In this article, we present a subset of the results of a study (11) that focused on developing a framework for improving IR training modalities for and with investigators in LMICs. The empirical findings presented here are meant to highlight IR's role in furthering knowledge translation efforts within LMICs. Our results indicate that the processes of communication and productive engagement of different stakeholder groups, the development of shared understanding and rendering evidence and concepts usable and valuable across differing professional domains are woven throughout the IR process. This view of knowledge translation requires a corresponding varied set of competencies; our findings indicate that prevailing modalities for IR capacity building in LMICs do not provide adequate opportunities for learners to develop them.

\section{IR Training Resources and Modalities}

The past decade has been marked by a surge in interest in IR. This trend is also accompanied by an ever-growing number of training programs specifically in IR - from short courses and boot camps to fully developed degree programs. Yet, the majority of these opportunities are offered primarily through North American or European institutions and remain out of the financial or practical reach of those from LMICs. One noteworthy exception to this is the library of training materials and short courses maintained by the Special Programme for Research and Training in Tropical Diseases (TDR), sponsored by the United Nations Children's Fund (UNICEF), the United Nations Development Programme (UNDP), the World Bank and the World Health Organization (WHO). TDR has developed a suite of self-study and hands-on IR training opportunities specifically for and with learners in LMICs, including a Massive Open Online Course (MOOC) for IR that has been running since 2018, with 2,600 participants meeting the requirements successfully and nearly 12,000 learners enrolled. Recent studies demonstrate its positive impact on learners' ability to carry out IR in their home contexts $(12,13)$. Nonetheless, for appropriate knowledge translation in support of NTD control programs, IR skillsets must continue to be improved among those conducting research and applying findings to practice.

As the dedicated UN think tank on global health, the United Nations University International Institute for Global Health (UNU-IIGH) partnered with TDR in November 2019 to better understand the IR training landscape for learners in LMICs. This study aimed to examine the unmet needs of IR learners to recommend a training approach that would better enable those involved in the knowledge translation process - namely researchers, health practitioners, policymakers, and the community - to utilize locally generated evidence to close the "know-do" gap. The findings described here cut across disease areas and are relevant for a range of health challenges encountered in LMICs.

\section{METHODS}

The study's methodological approach is summarized in Figure 1. The study was conducted in four main phases,- literature review, one-on-one interviews with key stakeholders, dialogues with IR training experts, and peer review of the findings. A modified Delphi method (14) was adopted in which each stage allowed for iteration and refinement of the concepts and approach to achieve a final consensus. This stepwise approach followed an iterative process of applying learning and feedback from prior phases to inform the development and validation of following materials. An in-depth explanation of the four-phase methodology is presented below. 


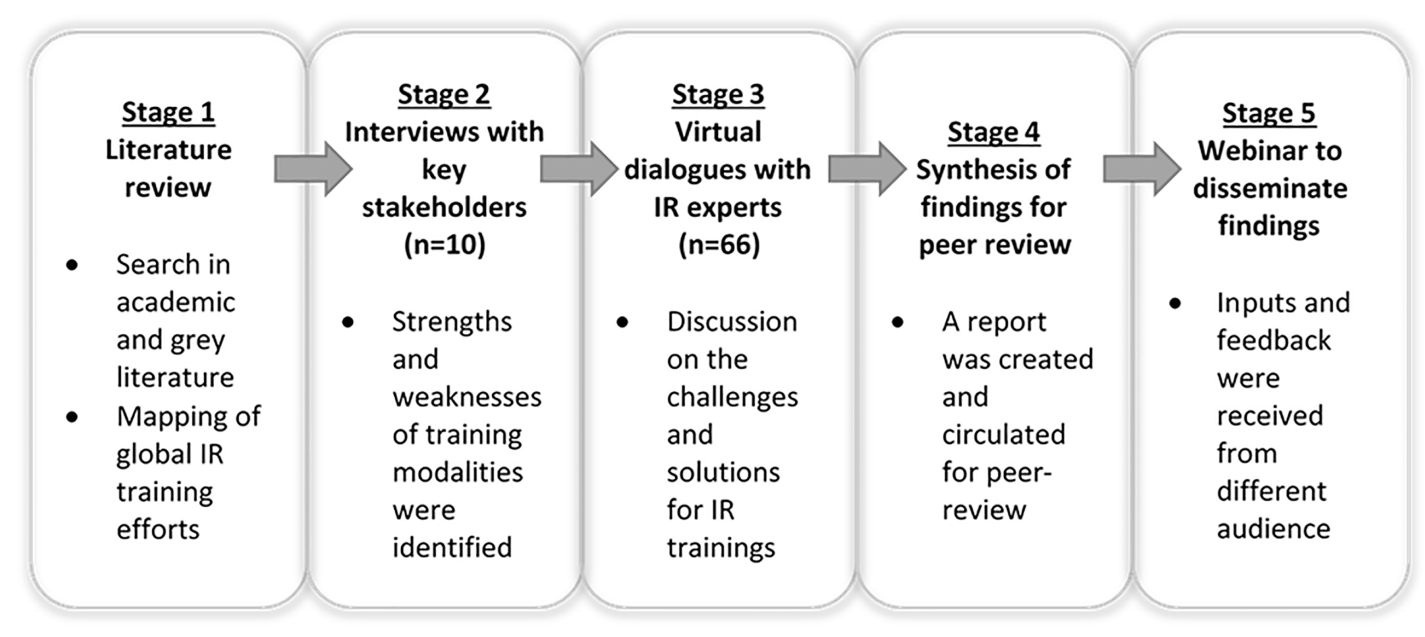

FIGURE 1 | Process for developing the IR training framework.

\section{Phase 1: Literature Review}

A review of the peer-reviewed and grey literature was undertaken in November 2019 as the first stage to explore recent and ongoing IR training efforts globally. We searched the Scopus database using the search terms "implementation research" or "implementation science" and "training". The search yielded 752 results. After screening abstracts for relevance, 19 papers were identified which focused on pertinent examples of IR training or highlighted relevant training concepts and needs. One additional paper from TDR authors that did not appear in the initial search was added manually. A snowball search strategy was employed to identify works from the grey literature using the Google search engine with "implementation research training" as the initial search term. A limited number of IR training examples were found through this approach. An Excel spreadsheet was used to record and categorize the articles, reports, and websites included in the literature review.

The literature review was guided by three primary objectives:1) identify the actors (including universities, institutes, and organizations) involved in IR training; 2) map the available training modalities (short courses, academic courses, and embedded project-based training); and 3) determine the training needs for IR investigators in LMICs. Initial literature review findings served as the basis for the topics covered in the semi-structured interviews with IR training experts.

\section{Phase 2: Individual, Semi-Structured, Expert Interviews}

Interview participants were identified through the author list of the reviewed publications and by soliciting nominations from key IR training stakeholders. An introductory email describing the purpose of the project and a request to schedule an online meeting was sent to potential participants in March and April 2020. A total of ten interviews with individuals from nine organizations were conducted; eight of the ten interview participants were from LMICs. Interviews with these critical stakeholders followed a pre-planned but modifiable guide. Interview analysis yielded insights into the strengths and weaknesses of current training programs and complemented the findings from the literature review. Interviewees were asked questions around the content and the modes of delivery of the capacity building initiatives they were involved in, their evolution, the profiles of learners and the barriers and factors that underlined teaching and learning. Interviewee suggestions and recommendations for improving the design of future IR training programs were also collated. The information from these one-on-one conversations, combined with the findings from the literature review, was used as the foundation for a virtual dialogue series conducted with a broader group of IR stakeholders.

The literature review and expert interviews identified three primary IR training modalities for researchers in LMICs, presented below. Participants in virtual discussions validated this typology.

- Academic courses delivered as a component of postgraduate curricula, e.g., Master of Public Health (MPH).

- Project-embedded training approaches seeking to improve the outcomes of a specific health initiative. These approaches usually involve training a local team in IR with input and support from international experts.

- Short, online professional courses, such as the TDR's IR MOOC, or face-to-face professional workshops and boot camps executed over a few days to several weeks.

\section{Phase 3: Virtual Dialogues With IR Practitioners, Capacity Strengthening Experts, and IR Training Participants}

Invitations were sent to IR experts identified through a review of the academic and grey literature, IR practitioners within the 
UNU-IIGH and TDR networks, and participants in TDR's IR MOOC. Individuals who accepted the invitation received a brief report highlighting the key findings from the literature review and expert interviews. The range of expertise involved in the virtual dialogues included the design and delivery of IR courses, adult learning, gender and health, and policymaking. Three recent TDR's IR MOOC participants were invited to attend so that learners' perspectives were also heard and considered

In September 2020, we organized two rounds of the dialogue series to accommodate the range of time zones represented for a total of four sessions. The four virtual dialogue sessions brought together 66 IR researchers, educators, and learners from 18 countries (40 of the 66 dialogue participants were from LMICs) (see Figure 2). This virtual dialogue series aimed to present and validate the findings from the literature review and expert interviews and surface omissions in our research. Discussions within the first session focused on the past successes and near-term developments in IR training and sought to identify persistent gaps in IR training (see Table 1). The second session focused on prioritizing the challenges identified in the first session and conceptualizing potential solutions to these obstacles. Each session lasted approximately two hours and included one or two breakout room discussions accompanied by plenary discussions. For the breakout room discussions, participants were divided into small groups of four to seven individuals, accompanied by a facilitator and a notetaker. Inputs and feedback were collected using a shared web-based platform enabling synchronous contributions from dialogue participants.

\section{Phase 4: Synthesis of Findings and Peer Review}

We synthesized the findings from the literature review, stakeholder interviews, and dialogues with experts and circulated our report for peer review. The goals of the synthesis were to twofold: 1) to highlight and reassess the assumptions behind existing training approaches and to identify the issues that existing approaches leave unaddressed and 2) to develop a cohesive framework for thinking and action among IR training stakeholders. The report highlighted pressing issues and provided examples of how potential solutions can be adapted according to the needs of different organizations, regions, and countries. Four reviewers with expertise in implementation research, health intervention delivery, health policy, monitoring and evaluation, and IR training delivery programs were engaged to review the synthesis report. The report was revised based on the comments from the reviewers and is now available online through the UNU-IIGH website (11). The study identified two types of challenges and opportunities for strengthening IR training in LMICs. The first set concerns pedagogical issues, namely what is being taught to whom and how. The second set relates to broader institutional features that hinder training and the scale-up of IR, such as raising awareness on the benefits and limitations of IR amongst decision-makers.

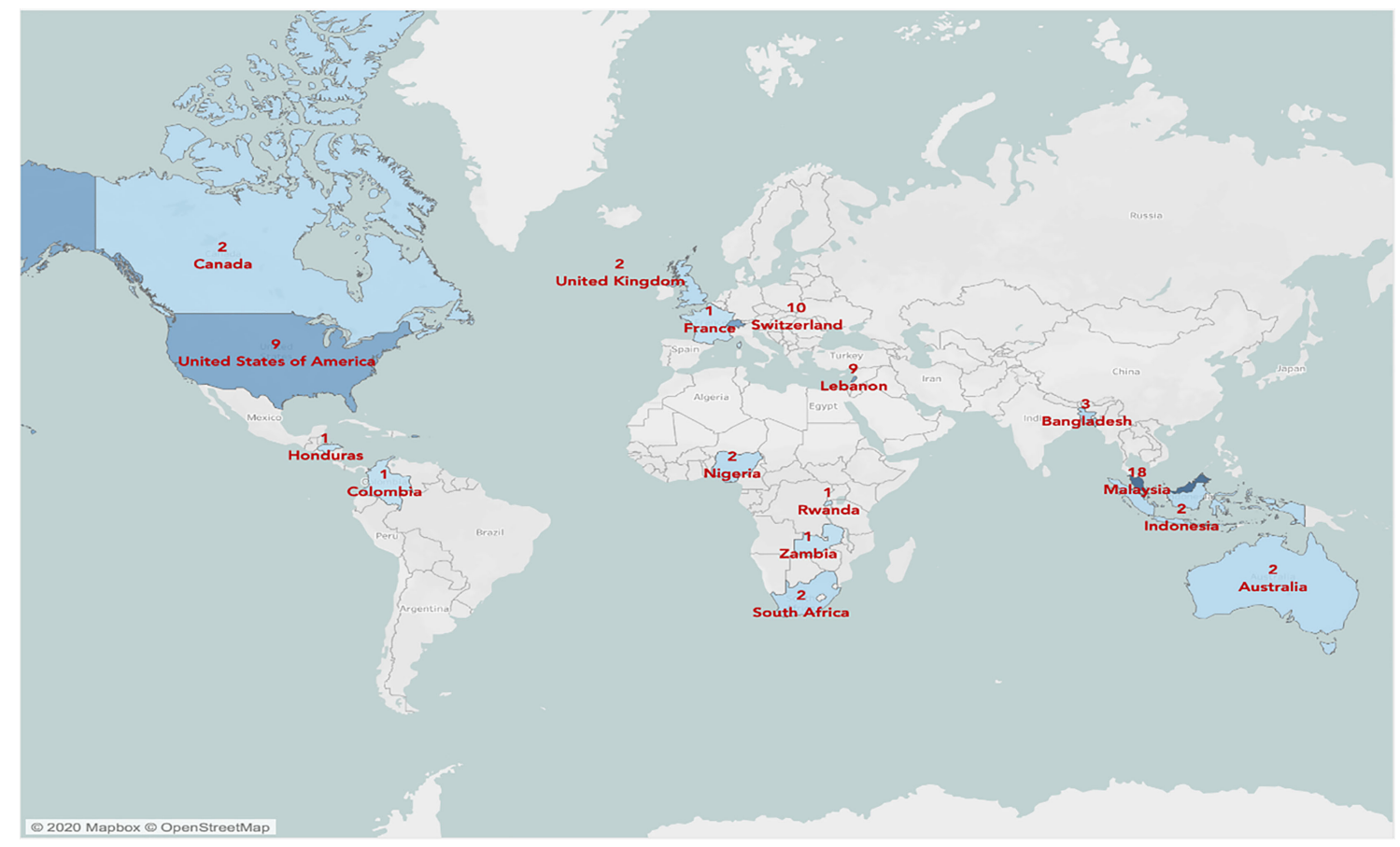

FIGURE 2 | Country distribution of IR experts who participated in the virtual dialogues. 
TABLE 1 | Discussion topics in the virtual dialogues.

\begin{tabular}{ll}
\hline Session 1 \\
\hline - & Reflections on the successes and challenges of IR capacity building in LMICs \\
- & Insights from former TDR's MOOC participants \\
- Discussion on integrating gender in IR training
\end{tabular}

The results presented in this article draw on the findings related to IR pedagogy and particularly to pedagogical issues that can enhance learners' ability to effectively translate different types of knowledge in a manner favored by the different communities of practice involved in an IR initiative.

\section{RESULTS}

\section{Pedagogical Challenges in IR Training for Supporting Multidisciplinary Collaboration}

Knowledge-translation is critical for multidisciplinary collaboration. This section presents three types of pedagogical challenges related to how existing training modalities build competencies for interdisciplinary collaboration. In our study, interdisciplinary collaboration involves teamwork across different disciplines and areas of expertise. In short, most available IR training approaches do not:

- Adequately factor in the diversity of IR learners in terms of epistemic and professional backgrounds.

- Provide opportunities for experiential, team-based learning.

- Acknowledge and support the different roles that individuals may assume as part of an IR team.

Interview and dialogue participants repeatedly emphasized that researchers and healthcare practitioners living and working in the communities where interventions are being rolled out have the most in-depth knowledge of the local context and the challenges encountered. These individuals are best placed to identify barriers and facilitators to implementation and help develop strategies to overcome bottlenecks encountered during scale-up. IR offers the tools needed to analyze the factors impeding a health system's ability to carry out a policy, program, or practice to its intended effect. These features mean that IR is intensely team-based and multidisciplinary. Most IR initiatives involve a combination of IR experts, implementors, health care professionals and practitioners engaged in the health intervention, and decision-makers from different levels of administration (15).

Expert interviewees and participants in the dialogue series indicated that IR learners have different epistemic backgrounds and first-hand experience with the health challenges that IR seeks to address. Some learners may lack an in-depth understanding of the context of an intervention. Others may be familiar with certain parts of an intervention, such as the on-ground delivery of a drug but may lack a holistic understanding of how the specific solution may relate to system-level bottlenecks. Our conversations with IR course designers and past trainees also revealed that many short course participants lack a grounding in
Session 2

- $\quad$ Reflections on IR training gaps

- Prioritization of the challenges with consideration of impact and effort

- Discussions of solutions for prioritized, high-impact challenges social science and health systems research. These learners may possess a solid clinical background or have prior experience in laboratory research but do not have a foundation in the core social science and policy-related research essential to IR.

Online and short professional courses examined through this study were generally not designed with this level of diversity in mind. As a result, many IR learners are tasked with learning too much information in too short a time - workshops often last only three or four days. For example those, lacking a background in social science research, such as health professionals and administrators, are asked to assimilate complex social science concepts and understand where and how to use them. Similarly, learners unfamiliar with the challenges associated with delivering efficacious health interventions in the 'real world', or with those being applied in a setting very different from their own, such as research professionals and university students, may not readily appreciate the difference IR can make in alleviating such difficulties. This aspect of the training was intensively discussed in our dialogue series.

Interviews with expert IR educators revealed that few existing training approaches cultivated the skills or the opportunities necessary to develop and function as part of an IR team. This deficit was reiterated and confirmed by participants in the virtual dialogue series organized for the study. Teamwork is an integral part of project-embedded training approaches by design. Still, workshops, boot camps, and other short courses typically overlook topics such as team building, communication, collaboration, and the development of a shared research language. The academic and short professional courses examined in our study were aimed primarily at developing individual capacity rather than collaborative skills. Interviewees confirmed that few existing training approaches cultivated the skills or the opportunities necessary to develop and function as part of an IR team. This deficit was reiterated and confirmed by participants in the virtual dialogue series. Several expert interviewees mentioned that they try to simulate the experience of working as part of a team by grouping participants in workshops according to their backgrounds and interests. Still, this task requires extra efforts and resources from the training facilitators and is said to yield mixed results.

Interview findings validated in virtual discussions show that most courses seek to strengthen capacity among researchers only. Current training curricula do not support the different roles that members of an IR team assume. For example, program implementors, decision-makers, and health practitioners' contributions to the IR team usually lay in their first-hand experience of the nature of the health challenges, the context of the intervention, and their network of connections that can enable access to external stakeholders and research sites. Consequently, these team members may not need to know the 
strengths and limitations of different research designs in detail or be involved in every data analysis stage to engage productively in the IR team $(16,17)$.

\section{Pedagogical Gaps and Opportunities in IR Training for Stakeholder and Community Engagement}

Interviewees and dialogue participants repeatedly noted that well-thought-out IR initiatives need to engage constructively with external stakeholders throughout the research process. These key stakeholders and community members may be gatekeepers of research sites or groups, expert advisors, advocates of the IR initiative occupying critical positions in the relevant parts of the health system, or influential members of communities that the intervention seeks to engage.

Stakeholder engagement was highlighted throughout our study as a topic that is touched upon but not fully addressed in most IR professional courses and project-embedded training programs. The literature review, expert interviewees, and participants in virtual dialogues spoke to the importance of addressing this gap concerning two categories of stakeholders: 1) decision-makers and 2) the broader communities of professionals and members of the public that the intervention concerns.

Participants in our virtual discussions pointed out that the term "decision-makers" is often used as a catch-all term and spent considerable time deliberating on who decision-makers are and how they can best support IR. It was agreed that this group's constitution would vary depending on the nature of the intervention but is likely to include people working at different levels of management and administration, from local community leaders and regional hospital directors to high-level government officials and national policymakers. Even individuals that are often seen as occupying lower positions of authority within health systems, such as community health workers, yield considerable power from the perspective of intervention beneficiaries. Decision-makers and healthcare practitioners on the IR research team can help refine who these individuals are and advise on their concerns, constraints, and priorities. This can help inform the research design, study questions, and project outputs and shape the team's communication strategy to increase the likelihood that key stakeholders will participate in and support the IR initiative.

Research participants are the second group of external stakeholders that are not adequately addressed in the typical IR course - apart from consent and privacy. Like decision-makers, research participants can belong to groups and may be included in the investigation in varying capacities, including a formal community engagement strategy (18). Given the nature of IR's execution, understanding what approaches to communication and engagement are best suited for different communities is a crucial subject in and of itself (19). However, as the experienced health researchers and course developers in our study noted, the skills needed to understand, map and navigate community dynamics, inequities, and gender-based and other social hierarchies are not typically instilled through IR courses. Success from this perspective means that considerations and acknowledgements of power dynamics are woven into the research process, supporting the accuracy and nuance of the results without reproducing or amplifying pre-existing inequities. Researchers can inadvertently reproduce existing inequities by favouring, for example, the perspectives of the more easily reachable members of the community or those who can express themselves freely in the spaces where consultations and inquiry occur. This process is more complex because formal titles and structures may not reflect social and political influence accurately, and power is often relational and fluid.

Experts in our study suggested that specific topics, such as gender lend themselves to explaining and illustrating the importance of power dynamics and can be an entry point to introducing more complex ideas, such as intersectionality. The expert interviewees and dialogue discussants also agreed that the knowledge and skills needed to engage different stakeholders successfully could not be learned solely in a classroom - virtual or otherwise. For example, a junior researcher may have taken a course on participatory poverty assessment but may not apply the lessons when community leaders are not in favour of a particular intervention. In our dialogue series, the IR practitioners and training experts highlighted the importance of developing these competencies through didactic and applied training.

\section{Pedagogical Challenges in IR Training for Translating Results Into Actions}

IR curricula fail to address the background and skills required to translate IR results into practical and actionable recommendations. Short professional courses and projectembedded approaches focus on research design, data collection and analysis, with little guidance on rendering research results practical and actionable for different audiences. This crucial stage in IR often occurs later in the research cycle once the necessary data have been collected and analyzed. However, seasoned researchers know that this process needs to begin much earlier in the investigation, with outlines for potential future actions and the communities that need to be informed in mind. These considerations further underline why decision-makers and health program implementors are core to a successful IR team and should be engaged throughout a project's lifecycle.

An interviewee indicated that many researchers, especially junior ones, struggle to package results in a manner that is readily understood by non-technical audiences. Even outputs aimed at informing policy audiences may fail to present the findings using engaging and non-technical language if those creating these outputs are not trained in this type of messaging. For example, a brief that outlines the changes needed to the delivery of an intervention may be helpful to individuals responsible for its onthe-ground implementation but may lack actionable advice around system-level constraints or funding needs relevant to high-level managers.

Dialogue participants agreed that IR findings could not be translated into meaningful recommendations without understanding the demands and requirements of the associated spheres of policy. No matter how scientifically sound an IR 
project is, the usefulness of its findings is diminished if researchers are unaware of what types of policy or programmatic action - and the avenues of influence - are needed to address identified implementation bottlenecks. A well-known case from the extensive research-to-policy literature concerns the importance of street-level bureaucrats (20), who are the actual people who implement a policy. Any policy initiative, be it small or large, is destined to fail if its creators do not take these decision makers' preferences, constraints, and motivations on the ground into account.

Participants in the dialogue series discussed at length the best way to involve critical stakeholder groups throughout the research process. It was agreed that the success of this process hinges on developing a relationship of trust and acknowledging and responding to the specific challenges and needs that these groups face.

The process of translating findings into action, therefore, depends on:

- Knowing whose actions (e.g., those of program administrators, community health workers, government decision-makers) carry the most significant weight and specifying the changes in outlook, policy, and practice given existing constraints and opportunities will likely bring about the desired outcome.

- Building this understanding into the research design and using the research process to refine it.

- Cultivate buy-in and advice from influential individuals to prepare early for research uptake throughout the study process.

The expert interviewees and dialogue participants reinforced the concept that successfully translating research into action depends on the IR team's degree of knowledge and skills associated with understanding, investigating, and navigating stakeholder dynamics.

\section{DISCUSSION AND CONCLUSIONS}

Our findings highlight the challenges prevalent in current training modalities that hinder the ability of learners to communicate, understand, and collaborate with different groups of stakeholders. This section presents the solutions developed through the project to address these shortcomings and elaborate on their importance for strengthening knowledge translation competencies in IR.

As discussed by interview and dialogue participants, current training approaches typically do not support the full range of learners who participate as an IR team member and lack the content needed to become proficient in meaningfully presenting key IR results to policy audiences. These omissions have prevailed because:

1. IR training programs are typically designed for researchers who are assumed to understand health systems research, not for learners unfamiliar with social science approaches or trainees for whom becoming involved in the minutiae of the research process may not be appropriate. Short courses and workshops, for example, often do not provide opportunities for self-paced learning, differentiated learning pathways for different IR team members, or opportunities to apply and refine their IR skills and knowledge. Therefore, investments in capacity building need to be guided by a clear understanding of the profile, needs and priorities of users of IR training resources and the factors that support or impede them in completing the training and applying their skills.

2. IR training offerings tend to focus on individual rather than team-based forms of learning. This shortfall is persistent even though IR is an intensive team-based and interdisciplinary undertaking whose success largely depends on the ability of the team to identify and mobilize different types of expertise and experience.

3. Courses do not thoroughly discuss the knowledge and skills necessary for community engagement and obtaining buy-in from decision-makers. Although existing curricula may highlight the importance of power dynamics, and some may suggest different strategies for community engagement, they do not impart the skills necessary for learners to map and negotiate these dynamics in practice.

4. Training programs do not impart the skills to translate research findings into actionable and practical recommendations to bring about the desired changes at different policy and practice levels. The success of this stage of IR depends on the IR team's ability to translate insights from their study into a language relevant to and understood by policymakers, health practitioners, program administrators, and the wider community. This advocacy process should ideally be planned for early in the IR cycle by including critical individuals within the IR team and obtaining constructive advice and buy-in from critical stakeholders.

Our study suggests that the first challenge can be addressed by enabling IR students to design their learning pathways, depending on their professional and epistemic backgrounds and priorities (21-23). This could be achieved by re-organizing existing materials into shorter units of learning (modules) and supplementing this library with complementary background materials from high-quality external sources to help different learners and IR team members develop relevant competencies (24). IR educators should create indicative pathways for certain learner groups to use as templates and adapt to their interests and needs. For example, someone with little or no grounding in social science may be recommended a pathway that starts with a unit on health systems research and qualitative inquiry.

Drawing on the insights from the interviews and dialogues, the second issue could be addressed by adopting a stronger focus on experiential team-based learning to instil learners with the skills needed to function as part of an interdisciplinary team (25). This experiential component is fundamental since teamwork involves several competencies, some of which cannot be taught in a classroom or workshop setting (virtual or physical). In the context of an IR study, for example, identifying and recruiting people with the necessary experience and expertise, 
troubleshooting the tensions arising from unavoidable miscommunications and the difficulties of the tasks at hand, and navigating cultural and professional dynamics whilst helping team members remain focused and motivated, are hard-won life skills.

The balance and sequencing between individual and team-based learning were explored in-depth in the interviews and dialogue series. Teams must learn to work together as a meaningful unit to carry out their collective task (26). Teams would benefit from individual members developing an understanding of IR through didactic training specific to their role and background. This could be supported by personalized learning journeys enacted in parallel to allow team members to understand IR's tools, processes, and vocabulary. Then the IR team could coalesce after individual competencies are formed. To effectively support team-based forms of learning, personalized learning journeys need to ensure team members develop a common vocabulary of IR concepts and tools while tailoring the content and competencies to each team members' background and role.

Expert interviewees noted that for experiential learning to be successful, it needs to be supplemented by reflection and expert advice. The discussions held through this study highlighted the importance of mentorship arrangements and the limited opportunities for mentorship among IR learners, particularly with regards to MOOC courses, which can experience participant enrollment in the thousands (27). Unfortunately, moderated online forums, a loose proxy for engagement with a research advisor, are difficult to tailor to the linguistic needs of large participant groups and are challenging for facilitators to engage learners actively in meaningful discussion (12). Matching IR teams under training with mentors from regional research institutions with shared interests could prove rewarding for both parties and serve as a basis for professional networking (28).

For addressing issue 3, we suggest that experiential learning may not be enough. This is because, whereas it may be safe to assume that many IR learners would have had the experience of working as part of a team in contexts other than IR, the ability to identify opportunities and barriers to participation and action, spot power divides and inequities, and skillfully navigate them are not usually part of regular academic or professional curricula. To this effect, we recommend that some of these topics, such as community engagement and intersectional gender analysis, are incorporated into current curricula. These topics will likely be most beneficial to learners if presented in didactic offerings and experiential-based learning. Where learning through on-theground intersectional gender analysis or community engagement is not feasible or appropriate, skills can be built through role-play exercises and dissection of high-quality case examples in a group format. Yet, expanding the content library of short courses, workshops, and MOOCs is not a simple matter, especially considering the quantity and density of materials already presented in these training programs. This consideration further exemplifies the utility of a modular approach to teaching - as new topics are integrated into training as stand-alone modules, they can be marked as compulsory or elective depending on the learner's background and allow past learners to explore additional concepts as new modules are created.
According to our online discussions, the most relevant solution to issue 4 hinges upon developing policy translation curricula specifically in the context of IR and by supporting opportunities for experiential learning beyond the research design, data collection, and analysis phases. There is a wealth of material from the researchto-policy literature that IR-specific approaches can build upon (29). Additionally, this area within IR offers decision-makers and practitioners opportunities to take the lead compared to other stages of the IR cycle, such as the methodological design phase. Enhancing training on these later phases of the IR cycle can help exemplify the importance of the policy and practice perspectives in IR and redress any imbalances that may exist due to IR training historically focusing on researchers.

As we have demonstrated, IR involves more than one knowledge translation process. For these processes to work, multiple forms of expertise and experience must be mobilized at different phases of the research cycle and considering the level of structural, systemic, and strategic support available to decision-makers (30). IR practitioners must develop the capacity to learn the specific dialects of policy and practice relevant to their initiatives. Policymakers and health program implementors wishing to address particular challenges and shape research questions must understand how IR can help meet their goals and best support an IR team. Our study revealed sets of skills and forms of knowledge translation need strengthening for the full potential of IR to be realized in LMICs.

\section{Limitations}

In light of our findings, there are several limitations worth mentioning. Initially, we designed this study as a progressive series of activities that would allow us to construct the revised IR training framework; this was not initially set up as a piece of empirical research. Regardless, the high-quality information gathered from experts and training participants and the resulting synthesis, in our opinion, should be disseminated widely to IR training designers and IR practitioners. We also acknowledge that the information reported here was collected from those with access to the technological resources necessary to participate in the online discussions and may not fully represent the opinions or experiences of those without this degree of connectivity.

\section{DATA AVAILABILITY STATEMENT}

The original contributions presented in the study are included in the article/supplementary material. Further inquiries can be directed to the corresponding authors.

\section{ETHICS STATEMENT}

Ethical review and approval was not required for the study on human participants in accordance with the local legislation and institutional requirements. Written informed consent for participation was not required for this study in accordance with the national legislation and the institutional requirements. 


\section{AUTHOR CONTRIBUTIONS}

All authors were involved in the conceptualization of the study. $\mathrm{MP}, \mathrm{EB}$, and SC prepared the first draft of the manuscript. All authors have read and approved the submitted version.

\section{FUNDING}

We acknowledge funding support from the Special Programme for Research and Training in Tropical Diseases (TDR), WHO.

\section{REFERENCES}

1. Mwandawiro C, Okoyo C, Kihara J, Simiyu E, Kepha S, Campbell SJ, et al. Results of a National School-Based Deworming Programme on SoilTransmitted Helminths Infections and Schistosomiasis in Kenya: 20122017. Parasit Vectors (2019) 12(1):76. doi: 10.1186/s13071-019-3322-1

2. Tchuem Tchuenté L-A, Rollinson D, Stothard JR, Molyneux D. Moving From Control to Elimination of Schistosomiasis in Sub-Saharan Africa: Time to Change and Adapt Strategies. Infect Dis Poverty (2017) 6(1):42. doi: 10.1186/ s40249-017-0256-8

3. Rollinson D, Knopp S, Levitz S, Stothard JR, Tchuem Tchuenté L-A, Garba A, et al. Time to Set the Agenda for Schistosomiasis Elimination. Acta Trop (2013) 128(2):423-40. doi: 10.1016/j.actatropica.2012.04.013

4. Diawara L, Traoré MO, Badji A, Bissan Y, Doumbia K, Goita SF, et al. Feasibility of Onchocerciasis Elimination With Ivermectin Treatment in Endemic Foci in Africa: First Evidence From Studies in Mali and Senegal. PloS Negl Trop Dis (2009) 3(7):e497. doi: 10.1371/journal.pntd.0000497

5. Molyneux DH, Savioli L, Engels D. Neglected Tropical Diseases: Progress Towards Addressing the Chronic Pandemic. Lancet (2017) 389(10066):31225. doi: 10.1016/S0140-6736(16)30171-4

6. Huang X, Deng X, Kou J, Liu X, Wang H, Cheng P, et al. Elimination of Lymphatic Filariasis in Shandong Province, China, 1957-2015. Vector Borne Zoonotic Dis (2020) 20(12):875-81. doi: 10.1089/vbz.2020.2624

7. Musanabaganwa C, Condo JU, Penkunas MJ, Pillai C, Mills E. Rwanda's Gains From Linking Science and Policy. Nature (2019) 565(7737):25-5. doi: 10.1038/d41586-018-07863-3

8. Sheikh K, Agyepong I, Jhalani M, Ammar W, Hafeez A, Pyakuryal S, et al. Learning Health Systems: An Empowering Agenda for Low-Income and Middle-Income Countries. Lancet (2020) 395(10223):476-7. doi: 10.1016/ S0140-6736(19)33134-4

9. Damschroder LJ, Aron DC, Keith RE, Kirsh SR, Alexander JA, Lowery JC. Fostering Implementation of Health Services Research Findings Into Practice: A Consolidated Framework for Advancing Implementation Science. Implement Sci (2009) 4(1):50. doi: 10.1186/1748-5908-4-50

10. Peters DH, Adam T, Alonge O, Agyepong IA, Tran N. Implementation Research: What It Is and How to Do It. BMJ (2013) 347:1-17. doi: 10.1136/ bmj.f6753

11. United Nations University International Institute for Global Health. Implementation Research Training Framework 2.0: Making the Whole Greater Than the Parts. Kuala Lumpur: United Nations University International Institute for Global Health (2021). Available at: http:// collections.unu.edu/view/UNU:8215.

12. Launois P, Maher D, Certain E, Ross B, Penkunas MJ. Implementation Research Training for Learners in Low- and Middle-Income Countries: Evaluating Behaviour Change After Participating in a Massive Open Online Course. Health Res Policy Sys (2021) 19(1):59. doi: 10.1186/s12961-02100703-3

13. Launois P, Allotey P, Reidpath D, Maher D, Certain E, Ross B. Lessons Learnt From a Professional Development MOOC: Engaging Culturally and Linguistically Diverse Learners From Low- and Middle-Income Countries. Eur J Open Distance E-Learn (2019) 22(2):1-16.

14. Humphrey-Murto S, Varpio L, Wood TJ, Gonsalves C, Ufholz L-A, Mascioli $\mathrm{K}$, et al. The Use of the Delphi and Other Consensus Group Methods in

\section{ACKNOWLEDGMENTS}

We thank all stakeholders who generously contributed their time, ideas, and insights to this project: the interviewees and workshop participants who made time to participate in the discussions that further contributed to our thinking; reviewers of the report: Dr Juliana Kagura, Dr Ana Bauman, Dr Yodi Mahendradhata and Dr Malabika Sarker for their constructive feedback; Dr Bella Ross for connecting us with stakeholders; as well as all facilitators and notetakers for their support in the workshops.

Medical Education Research: A Review. Acad Med (2017) 92(10):1491-8. doi: 10.1097/ACM.0000000000001812

15. Sturke R, Harmston C, Simonds RJ, Mofenson LM, Siberry GK, Watts DH et al. A Multi-Disciplinary Approach to Implementation Science: The NIHPEPFAR PMTCT Implementation Science Alliance. JAIDS J Acquired Immune Defic Syndr (2014) 67:S163. doi: 10.1097/QAI.0000000000000323

16. Bauer MS, Damschroder L, Hagedorn H, Smith J, Kilbourne AM. An Introduction to Implementation Science for the Non-Specialist. BMC Psychol (2015) 3(1):1-12. doi: 10.1186/s40359-015-0089-9

17. Tabak RG, Padek MM, Kerner JF, Stange KC, Proctor EK, Dobbins MJ, et al. Dissemination and Implementation Science Training Needs: Insights From Practitioners and Researchers. Am J Prev Med (2017) 52(3 Suppl 3):S322-9. doi: 10.1016/j.amepre.2016.10.005

18. O’Mara-Eves A, Brunton G, McDaid D, Oliver S, Kavanagh J, Jamal F, et al. Community Engagement to Reduce Inequalities in Health: A Systematic Review, Meta-Analysis and Economic Analysis. Public Health Res (2013) 1 (4):1-526. doi: 10.3310/phr01040

19. Glandon D, Paina L, Alonge O, Peters DH, Bennett S. 10 Best Resources for Community Engagement in Implementation Research. Health Policy Plann (2017) 32(10):1457-65. doi: 10.1093/heapol/czx123

20. Lipsky M. Street-Level Bureaucracy, 30th Anniversary Edition: Dilemmas of the Individual in Public Service. New York: Russell Sage Foundation (1980). $300 \mathrm{p}$.

21. McGivney V. Understanding Persistence in Adult Learning. Open Learn: J Open Distance E-Learn (2004) 19(1):33-46. doi: 10.1080/0268051042000177836

22. Sztajn P, Confrey J, Wilson PH, Edgington C. Learning Trajectory Based Instruction: Toward a Theory of Teaching. Educ Res (2012) 41(5):147-56. doi: 10.3102/0013189X12442801

23. Guàrdia L, Maina M, Sangrà A. MOOC Design Principles.A Pedagogical Approach From the Learner's Perspective. e-Learning J (2013) 33:6.

24. Alonge O, Rao A, Kalbarczyk A, Maher D, Marulanda ERG, Sarker M, et al. Developing a Framework of Core Competencies in Implementation Research for Low/Middle-Income Countries. BMJ Global Health (2019) 4(5):e001747. doi: 10.1136/bmjgh-2019-001747

25. Michaelsen LK, Sweet M. The Essential Elements of Team-Based Learning. New Dir Teach Learn (2008) 2008(116):7-27. doi: 10.1002/tl.330

26. Kayes AB, Kayes DC, Kolb DA. Experiential Learning in Teams. Simul Gaming (2005) 36(3):330-54. doi: 10.1177/1046878105279012

27. Leon Urrutia M, White S, Dickens K, White S. Mentoring at Scale: MOOC Mentor Interventions Towards a Connected Learning Community (2015). Available at: https://eprints.soton.ac.uk/373982/.

28. Penkunas MJ, Chong SY, Rhule ELM, Berdou E, Allotey P. Designing the Next Generation of Implementation Research Training for Learners in Low- and Middle-Income Countries. Global Health (2021) 17(1):63. doi: 10.1186/ s12992-021-00714-3

29. Kalbarczyk A, Rodriguez DC, Mahendradhata Y, Sarker M, Seme A, Majumdar $\mathrm{P}$, et al. Barriers and Facilitators to Knowledge Translation Activities Within Academic Institutions in Low- and Middle-Income Countries. Health Policy Plann (2021) 36(5):728-39. doi: 10.1093/heapol/czaa188

30. Mahendradhata Y, Kalbarczyk A. Prioritizing Knowledge Translation in Lowand Middle-Income Countries to Support Pandemic Response and Preparedness. Health Res Policy Sys (2021) 19(1):5. doi: 10.1186/s12961020-00670-1 
Author Disclaimer: The author PL is a staff member of the World Health Organization. The author alone is responsible for the views expressed in this publication and they do not necessarily represent the views, decisions or policies of the World Health Organization.

Conflict of Interest: EB was employed by Data Yarns Research.

The remaining authors declare that the research was conducted in the absence of any commercial or financial relationships that could be construed as a potential conflict of interest.

Publisher's Note: All claims expressed in this article are solely those of the authors and do not necessarily represent those of their affiliated organizations, or those of the publisher, the editors and the reviewers. Any product that may be evaluated in this article, or claim that may be made by its manufacturer, is not guaranteed or endorsed by the publisher.

(c) World Health Organization 2020. Licensee Frontiers Media SA. This is an open access article distributed under the terms of the Creative Commons Attribution IGO License (http://creativecommons.org/licenses/by/3.0/igo/legalcode), which permits unrestricted use, adaptation (including derivative works), distribution, and reproduction in any medium, provided the original work is properly cited. In any reproduction or adaptation of this article there should not be any suggestion that WHO or this article endorse any specific organization or products. The use of the WHO logo is not permitted. This notice should be preserved along with the article's original URL. 